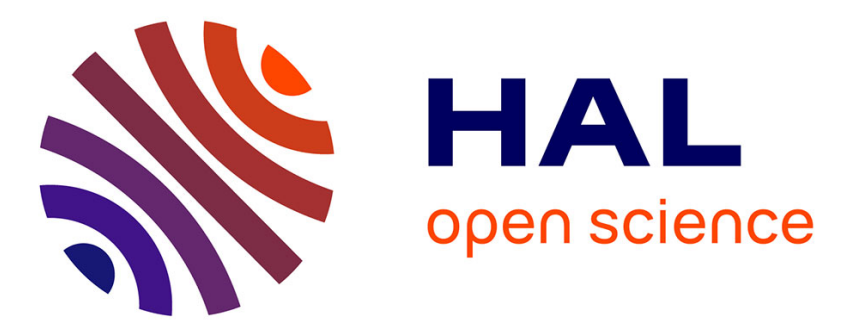

\title{
Highly reproducible, hysteresis-free, flexible strain sensors by inkjet printing of carbon nanotubes
}

Fulvio Michelis, Laurence Bodelot, Yvan Bonnassieux, Bérengère Lebental

\section{To cite this version:}

Fulvio Michelis, Laurence Bodelot, Yvan Bonnassieux, Bérengère Lebental. Highly reproducible, hysteresis-free, flexible strain sensors by inkjet printing of carbon nanotubes. Carbon, 2015, 95, pp 1020-1026. 10.1016/j.carbon.2015.08.103 . hal-01217246

\section{HAL Id: hal-01217246 \\ https://hal.science/hal-01217246}

Submitted on 19 Oct 2015

HAL is a multi-disciplinary open access archive for the deposit and dissemination of scientific research documents, whether they are published or not. The documents may come from teaching and research institutions in France or abroad, or from public or private research centers.
L'archive ouverte pluridisciplinaire HAL, est destinée au dépôt et à la diffusion de documents scientifiques de niveau recherche, publiés ou non, émanant des établissements d'enseignement et de recherche français ou étrangers, des laboratoires publics ou privés. 


\title{
Highly reproducible, hysteresis-free, flexible strain sensors by inkjet printing of carbon nanotubes
}

\author{
Fulvio Michelis ${ }^{1,2}$, Laurence Bodelot ${ }^{3}$, Yvan Bonnassieux $^{2}$, Bérengère Lebental ${ }^{1,2}{ }^{*}$ \\ ${ }^{1}$ Université Paris-Est, IFSTTAR, COSYS/LISIS, Paris, France \\ ${ }^{2}$ Laboratoire de Physique des Interfaces et Couches Minces (LPICM), UMR 7647, \\ Ecole Polytechnique-CNRS, Palaiseau, France \\ ${ }^{3}$ Laboratoire de Mécanique des Solides (LMS), UMR 7649, Ecole Polytechnique-CNRS, Palaiseau, France
}

\begin{abstract}
In order to build upon the exceptional interest for flexible sensors based on carbon nanotube networks (CNNs), the field requires high device-to-device reproducibility. Inkjet printing has provided outstanding results for flexible ohmic sensors in terms of reproducibility of their resistance. However, the reproducibility of the sensitivity, the most critical parameter for sensing application, has been only marginally assessed. In the present paper, CNN-based resistive strain sensors fabricated by inkjet-printing on flexible Ethylene Tetrafluoroethylene (EFTE) sheets are presented. The variability on the device initial resistance is studied for 5 different batches of sensors from 3 to 72 devices each. The variability ranges between $8.4 \%$ and $43 \%$ depending on the size of the batches, with a $20 \%$ average. An 8 -device batch with $15 \%$ variability on initial resistance is further studied for variability on the strain and thermal sensitivity. Standard deviation values are found to be as low as $16 \%$ on the strain sensitivity and $8 \%$ on the temperature sensitivity. Moreover, the devices are hysteresis free, a rare achievement for CNT strain sensors on plastics.
\end{abstract}

\section{Introduction}

Owing to their very large specific surface area [1], carbon nanotubes (CNTs) have been of utmost interest for sensing applications since the early days of CNT research [2]. Ohmic or transistor devices using CNTs deposited or grown on rigid substrates have demonstrated exceptional sensitivity to their environment, leading to various examples of analytical (humidity, $\mathrm{pH}$, gas, chemical or biological species) [3], mechanical (strain, pressure) [4] or radiation (thermal or infrared, UV) [5] sensors. Following the quick rise of CNT-based flexible electronics [6], a wide range of flexible CNT sensors was proposed [7], with the goal of providing the next generation of wearable devices [8] for human welfare monitoring or wireless sensor networks [9] for infrastructure [10] or environmental

\footnotetext{
*Corresponding author. Tel: +33 1816681 18. E-mail: berengere.lebental@ifsttar.fr (Bérengère Lebental)
} 
monitoring [11]. With their mechanical robustness (high Young's modulus, low bending rigidity, low buckling properties, high tensile strength [2] [12]) flexible CNT sensors are expected to provide longlasting, reliable devices compatible with industrial requirements.

The active component of these sensors most often consists of wet-processed carbon nanotube networks (CNNs) [13]. CNNs are films of randomly or partially organized CNTs and their thickness ranges from a few tens of nanometers to a few tens of micrometers. Their fabrication methods include filtration and extraction of buckypaper [14], spray coating [15], layer-by-layer assembly [10] as well as contact (roll-to-roll [16] or transfer-based [17]) and non-contact (aerosol [18] or inkjet [19]) printing techniques. Except for the buckypaper approach, all these methods rely on deposition over a substrate. Studies have yielded strain gauges [18] [20], gas sensors [21], photodetectors [22] and chemistors [23]. Table 1 in Supplementary information 1 provides an extensive study of the state of the art of CNN rigid and flexible sensors.

A long-standing, acute challenge for industrial applications of flexible CNN sensors lies in their low device-to-device reproducibility [24]. Reproducibility specifically addresses two factors, firstly, the standard deviation in initial device resistance, secondly, the standard deviation in device sensitivity. Device-to-device variability has been discussed repeatedly with regard to CNN-based flexible resistors [18] and transistors [25] and various causes have been reported, including liquid-phase dispersion issues of CNTs [26], variability in CNT properties within a batch (semiconducting versus metallic, diameter, length, sidewall defects) [24], quality of the CNT-to-electrode contacts or surface roughness of the flexible substrates [13].

In spite of this, reports on CNN sensors (either rigid of flexible) rarely provide standard deviation on the resistance level (see Table 1, SI1). Lowest reported standard deviations have been achieved via inkjet-printing: Benchirouf et al. [27] reports 3.5\% standard deviation on the resistance level of 3device-batches (strain sensors) while Lesch et al. [19] reports $7 \%$ and $20 \%$ standard deviation on the two fitting parameters of the amperometric cycle of antioxidant power sensors, for a 6-device batch.

Regarding to studying variability on sensitivity, Lesch et al. [27] and Karimov et al [28] provide the response of respectively 2 sets and 1 set of 2 devices. Kim et al. [29] , Mirica et al [30], Takeo et al [30] compare respectively 4, 3 and 3 sensors from the same batch, but provide no standard deviation on the sensitivity. Beyond this, Li et al. [31] provide the standard deviation on the benchmarking parameters (slope/sensitivity and Y-intercept, respectively $7 \%$ and $25 \%$ ) of a 3-device batch of gas sensors on SiO2. Finally, the most statistically relevant results stem from Lee et al. [32]. Lee et al. provide the full dispersion plot of the sensitivity of a batch of 21 sensors on $\mathrm{SiO} 2$, which spans two orders of magnitude. No comparable study is presently available for devices on flexible sensors. 
In the present paper, we provide a detailed study on resistance and sensitivity variability in batchfabricated inkjet-printed CNN-based flexible sensors. The study focuses on strain sensors based on multi-walled CNTs (MWCNTs) printed on Ethylene Tetrafluoroethylene (EFTE) sheets. After describing the fabrication process, we prove the high device-to-device reproducibility in terms of strain and temperature sensitivity. We also present the sensing performance, including exceptional cyclability and hysteresis-free operation.

\section{Methods}

\subsection{Materials}

MWCNTs Graphistrenth C100 are purchased from Arkema. The solvents 1,2-Dichlorobenzene (Dichlorobenzene hereafter), Acetone and Methanol and the surfactant Sodium Dodecyl Benzene Sulphonate (SDBS) are purchased from Sigma-Aldrich. The substrate is a $0.125 \mathrm{~mm}$ thick, $30 \mathrm{~cm}$ by 30 $\mathrm{cm}$ foil of Ethylene Tetrafluoroethylene (ETFE) with $90 \mathrm{~nm}$ roughness (calculated as in [33], see formula in Supplementary Information (SI) 1) supplied by Goodfellow.

\subsection{Carbon nanotube ink preparation}

The MWCNTs are dispersed in dichlorobenzene at $0.02 \mathrm{wt} . \%$ using an ultrasonic probe (Bioblock Scientific VibraCell 75043) operated at $150 \mathrm{~W}$ for $20 \mathrm{~min}$ followed by centrifugation at $10 \mathrm{kG}$ for $4 \mathrm{~h}$. In order to increase ink-wettability on ETFE and improve homogeneity of the deposition, SDBS at $0.3 \mathrm{wt} . \%$ is added to the supernatant using a $20 \mathrm{~min}$ sonication bath (see details in SI2) [13]. The resulting dispersion, called ink in the rest of the paper, is stable for over 3 months.

\subsection{Device fabrication}

The device structure is shown in Figure $1 \mathrm{a}$ and $\mathrm{b}$. The ETFE foils are first cleaned with acetone and dried under nitrogen flow. Pairs of $100 \mathrm{~nm}$ thin, $5 \mathrm{~mm} \times 5 \mathrm{~mm}$ gold electrodes spaced by $7 \mathrm{~mm}$ are deposited using thermal evaporation under vacuum ( $\left.10^{-7} \mathrm{mbar}\right)$. The MWCNTs are then deposited by inkjet printing using the customizable Dimatix Material Inkjet Printer 2800 with DMP-11601 cartridges. The cartridge is kept at room temperature during printing and the substrate is heated to $55{ }^{\circ} \mathrm{C}$. The printing pattern is a $17 \mathrm{~mm} \times 5 \mathrm{~mm}$ rectangle positioned to fully cover both electrodes of each device, in order to ensure proper electrical contact. Several layers can be printed successively depending on the properties targeted for the devices. Residual dichlorobenzene and surfactant are rinsed away by immersion and slight agitation in methanol and acetone for $8 \mathrm{~s}$ each, followed by drying under nitrogen flow.

The number of layers and the rinsing periodicity strongly impact the final device resistance (see details in SI.3). We optimized these parameters in order to reach device resistances below $1 \mathrm{M} \Omega$ 
(threshold value required for compatibility with commercial acquisition cards; see next section 2.4) while keeping the fabrication time manageable. Devices presented here are obtained from 20 printed layers rinsed every two layers. They are produced by batches of either 8 or 144 sensors on ETFE foils (Figure 1c). The time required for the production of 8 or 144 devices with 20 layers and rinsing every 2 layers is 3 and 5 days respectively.
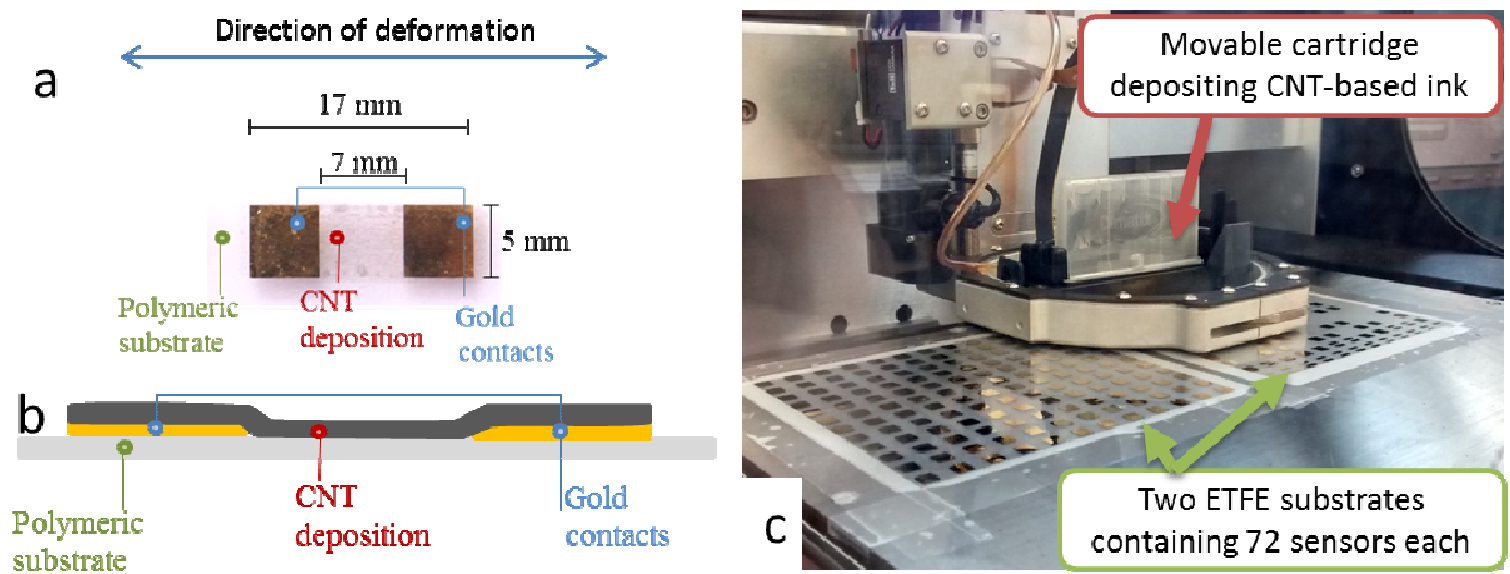

Figure 1 a) Image of a CNT-based device printed on ETFE (top view) with device size and direction of deformation. b) Cross-sectional diagram of the device. c) Ink-jet printing of a 144-device batch on side-by-side ETFE foils. Each substrate contains 72 sensors.

\subsection{Physical and electrical characterizations}

The devices are observed via optical and scanning electron microscopy SEM (Hitachi S 4800). The deposition thickness is measured via profilometry (Dektak 150). For resistance measurements, electrical contacting is achieved by gluing thin copper wires to the gold electrodes with silver paste. The resistance is measured in a 4-probe configuration to limit the impact of contact resistances: a Keithley 2612 source measurement unit imposes a constant, continuous current in the $\mu \mathrm{A}$ range (from 4 to $7 \mu \mathrm{A}$ ) while a National Instrument N19212 acquisition card measures the output voltage at a frequency of $6.7 \mathrm{~Hz}$. All electrical characterizations are performed within a Faraday cage providing electromagnetic shielding.

\subsection{Strain sensing}

To characterize the devices as strain gauges, variations in resistance are measured while applying longitudinal deformation to the substrate (deformations applied along the main axis, see Figure 1a). Deformations are applied by gluing the two opposite sides of a substrate to two clamps mounted on force-controlled motors (Figure 2). Forces up to $8 \mathrm{~N}$ are applied. The force is measured using Althen ALF328 load cells. A CCD camera is used to determine the resulting deformation of the substrate: the 
displacement of surface patterns is tracked during deformation and then converted into strain. Strain levels reach up to $2500 \mu \varepsilon$.

Devices are characterized either separately or by series of 4 sensors on the same substrate. During characterization of the piezoresistive response, the temperature is not controlled but is monitored by a thermocouple.

\subsection{Temperature sensitivity}

The temperature sensitivity of the resistance is also measured. The devices are placed on a hot plate controlled by the Thermoelectric Temperature Controller LFI 3751 by Wavelength Electronics. The resistance is measured with a Keithley S4200 using a 4-probe configuration. During temperature characterization, the samples are positioned in a shielded environment under nitrogen flow to ensure that relative humidity remains constant at $0.8 \%$ during the duration of the measurement.

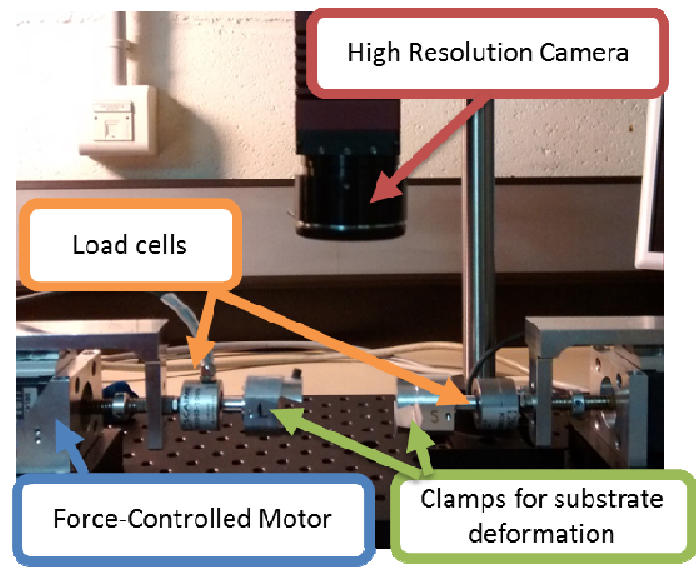

Figure 2 Extensometric bench used for electromechanical characterization.

\section{Results and interpretation}

\subsection{Morphology of the carbon nanotube network}

SEM images of a single layer deposition (Figure 3a) show that CNTs overlap each over and are spread randomly on the surface, with a surface density of $240 \mathrm{CNT} / \mu \mathrm{m}^{2}$ as obtained by visual counting on high resolution images (see SI5 for more details). Numerous micrometric holes are observed, probably due to loss of material during rinsing. When increasing the number of layers, the homogeneity of the deposition improves and the surface coverage increases. At 20 depositions, the surface is entirely covered (Figure $3 b$ and $c$ ).

Up to 20 layers, the thickness of the deposition increases quadratically for each additional layer and reaches $1100 \mathrm{~nm}$ for a 20-layer deposition (Figure 3d). As expected from the SEM images, the film surface is very rough. The roughness (error bars in Figure $3 \mathrm{~d}$ ) increases with the number of layers, 
from $110 \mathrm{~nm}$ for 3 layers up to $720 \mathrm{~nm}$ for 20 layers. The substrate itself contributes to the overall roughness by only $90 \mathrm{~nm}$. The faster than linear rise of the thickness (clear despite the large roughness/error bars) suggests that the film porosity increases with increasing number of layers, probably due to the imperfect filling of the holes (Figure 3a).
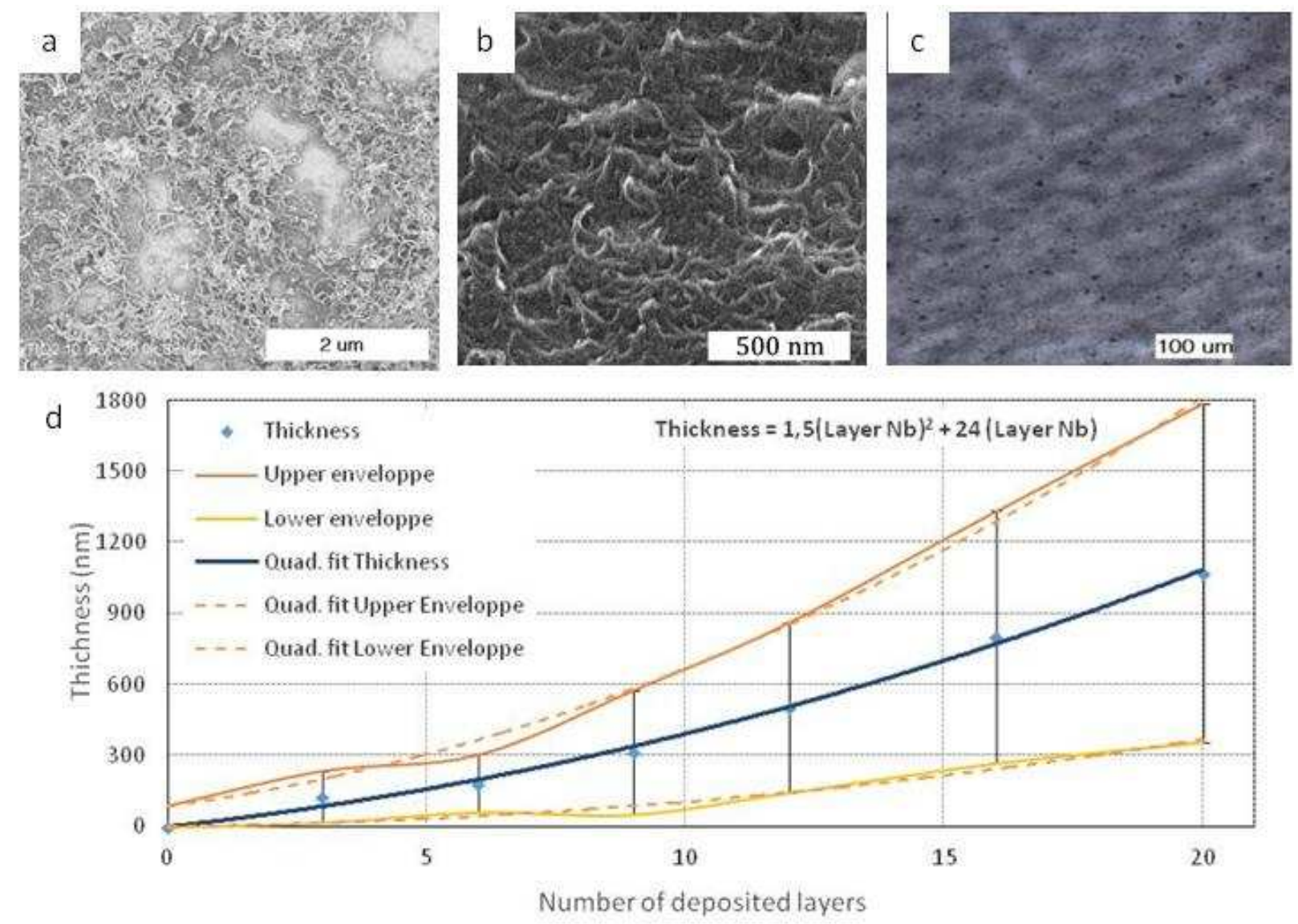

Figure 3 a) SEM image of 1-layer deposition. Micrometric holes are observed in the layer; they are attributed to loss of matter during rinsing. b) SEM image of 20-layer deposition. A uniform coverage can be observed. c) Optical microscopy image of a 20-layer deposition, showing homogeneity and uniformity at a micro scale. d) Thickness of the deposition with respect to number of layers. The rise is roughly quadratic. The error bars represent the surface roughness (the error bar for 0 layer is the roughness of the substrate, $90 \mathrm{~nm}$ ). They are used to define the envelope of the thickness curve. Both upper and lower envelopes also rise quadratically.

\subsection{Device resistance}

A single layer has a resistance in the $G \Omega$ range, too high for sensing applications. As expected, the resistance decreases as the number of layers increases, in accordance with the literature on CNTs percolated networks [34]. It reaches the $1 \mathrm{M} \Omega$ range at 15 layers, the $150 \mathrm{k} \Omega$ range at 20 layers and the $100 \mathrm{k} \Omega$ range at 30 layers (Figure 4). The lowest resistance reached is $104 \mathrm{k} \Omega(87 \mathrm{k} \Omega / \mathrm{sq}$ ) for a 30 layer deposition. This range of magnitude of resistance, though quite high compared to CNN-based inkjet-printed flexible transparent conducting layers [35], is fully compatible with sensing 
applications. The decrease of resistance as a function of the thickness is much faster than for homogeneous thin films (see for instance Fuchs model [36]). It is attributed to the decrease of the roughness-to-thickness ratio of the films when the number of layer increases (as predicted by Namba model for rough thin films [37]).

Standard deviations for 3- to 72-device-batches of 20-layer devices were found to range from $8.4 \%$ (3 devics) to $43 \%$ (72 devices) with an average at 20\%. (Details on the standard deviations of the different batches are provided in SI6). The larger standard deviation for the large batch is attributed to a degraded homogeneity in the gold deposition over the full area of the ETFE sheet. For smaller batches ( 3 to 8 devices; standard deviations ranging from $8.4 \%$ to $18 \%$ ), the differences in variability are attributed to slight variations in the ink quality.

In the rest of the paper, we discuss a 20-layer, 8-sensor batch with average resistance $156 \mathrm{k} \Omega$ and $15 \%(23 \mathrm{k} \Omega)$ standard deviation. This batch was selected as it featured the lowest average resistance and was of sufficient size for further sensitivity analysis. The batch with lowest standard deviation had only 3 devices and thus appeared too small for standard deviation calculation.

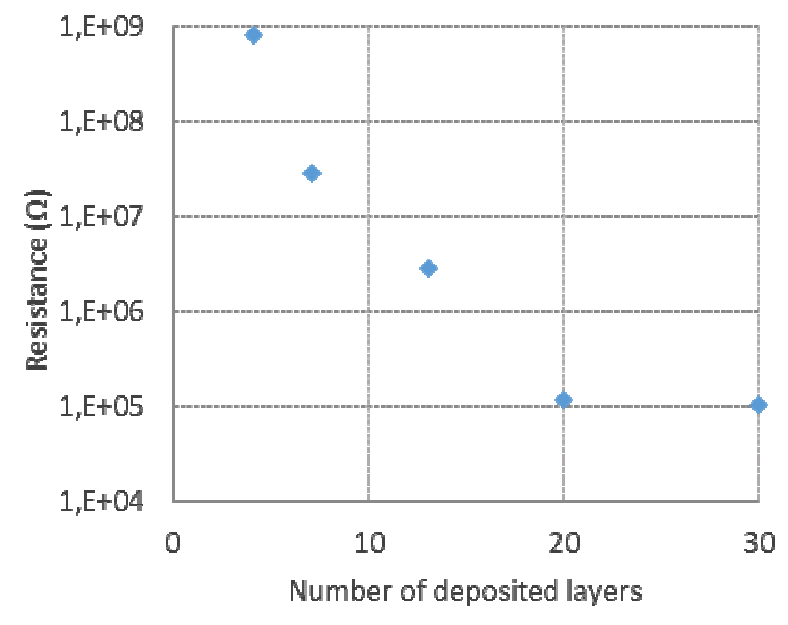

Figure 4 Resistance of the deposition with respect to number of layers.

\subsection{Reproducibility of strain sensing performances}

As expected from the literature on CNNs strain sensitivity [15] [18], the resistance of the devices varies when they are subjected to longitudinal strains of up to $2500 \mu \varepsilon$ (Figure 1a). In this range of strain, the strain-stress relationship of the substrate is linear (see SI7 for details on the mechanical properties of the film). For large strains up to $2500 \mu \varepsilon$ (1 $\mu \varepsilon$ strain corresponds to an extension $\Delta \mathrm{L}$ of the device initial length $\mathrm{L}$ by $\Delta \mathrm{L}=10^{-6} \mathrm{~L}$ ), the resistance increases quadratically with strain, reaching up to $0.6 \%$ (about $900 \Omega$ ) of the initial resistance (Figure 5a), in accordance with [38] (see SI 8). The resistance variation measured on the gold electrodes when they are subjected to strain of up to 
$2500 \mu \varepsilon$ is $10 \Omega$, over 10 times smaller than the resistance variation for the whole sensing element. Consequently, we can deduce that the piezoresistive effect is not due to the electrodes only.

All devices feature a linear behavior in the small strain regime. The linear range exceeds $400 \mu \varepsilon$ for all the devices. $75 \%$ have a linear range of over $600 \mu \varepsilon$ and $50 \%$ above $800 \mu \varepsilon$. The strain sensitivity, also called gauge factor (GF), is defined as the slope of $\Delta R / R$ vs $\varepsilon$ curve in the linear regime. It is found to be $0.90 \pm 0.14$ for an 8-device batch, corresponding to $16 \%$ standard deviation on the GF. The 0.9 GF value is the only reported GF for printed CNT resistive strain sensors [39]. This is the first quantitative evaluation of the variability in sensitivity for batch-produced CNT-based flexible sensors.

This GF value is comparable to the GF of commercially available strain gauges (between 1 and 2), though the standard deviation on the resistance and on the gauge factor are still higher than the dispersion reported in the datasheets of commercial devices (respectively $2 \%$ and $5 \%$ standard deviation on the resistance and on the GF) [40] [41]. Much higher GF values can be achieved in devices with oriented CNT [8] or closer to the CNN's percolation threshold, including buckypaperbased devices (for instance [42]). However operation close to the percolation threshold has a detrimental impact on device-to-device reproducibility [18].

\subsection{Reproducibility of temperature sensitivity}

Temperature is known to strongly influence both the conductivity of CNNs [43] and the mechanical response of polymer foils [44]. Hence, we studied the dependence of the devices on temperature (Figure $5 b$ ). The results show that the resistance decreases linearly with temperature, in accordance with other studies [43] [45]. The temperature sensitivity, defined as the slope of the $\Delta R / R$ versus temperature curve, is equal to $-1.0 \times 10^{-3} \mathrm{~K}^{-1}$. This coefficient is comparable to those published in [43]and [46] for CNT devices fabricated respectively on silicon and PET. The thermal sensitivity cannot be explained by the thermal expansion of the substrate $\left(0.9-1.7 \times 10^{-4} \mathrm{~K}^{-1}\right)$ [47]), as the effect would be a positive thermal sensitivity in the range of $+0.8-1.5 \times 10^{-4} \mathrm{~K}^{-1}$. Dehghani et al. [43] proposes that the thermal sensitivity is mostly due to thermal variation in the CNT resistivity.

The standard deviation in the temperature sensitivity is as low as $8 \%$ over 7 devices, even lower than the standard deviation over resistance (15\%) and over gauge factor $(16 \%)$. The possibility of compensating for temperature is critical for future applications of this strain gauge. 

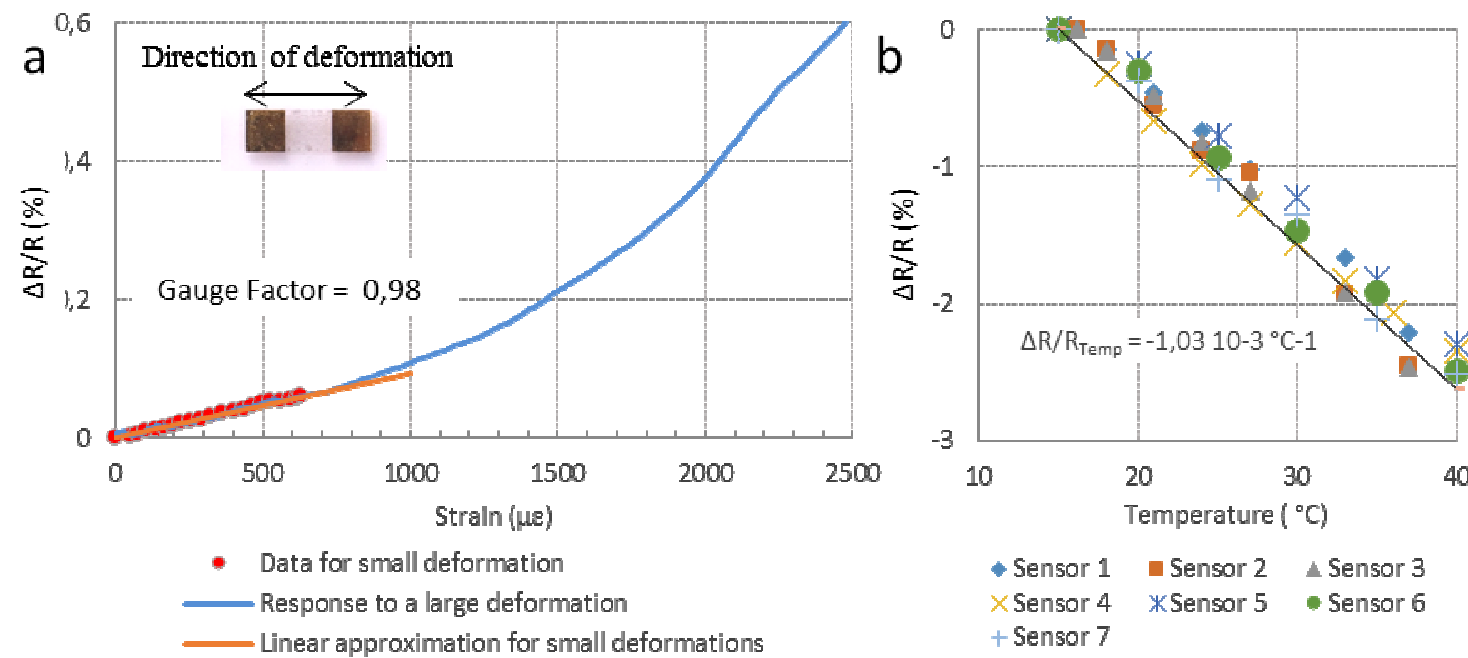

Figure 5 a) Resistance variation with respect to strain. The linear regime reaches up to $700 \mu \varepsilon$ for this device. The gauge factor of the device presented here is 0,98 . Overall, the response up to large strains is roughly quadratic.b) Linear dependence of resistance on temperature. The temperature sensitivity is equal to $-1.0 \times 10^{-3} \mathrm{~K}^{-1}$.

\subsection{Response time}

We assessed the device response time, defined here as the time to reach $95 \%$ of the expected value once the target strain is reached (Figure $6 a$ and $b$ ). The strain is increased linearly over $5 \mathrm{~s}$ to reach its target (see Figure 6a). The response time was found to be $3.6 \mathrm{~s}$ for a $500 \mu \varepsilon$ strain (within the linear regime of the device). This suggests that the devices should preferably be used for static or quasistatic applications (frequencies well below $0.3 \mathrm{~Hz} /$ period well over $3.6 \mathrm{~s}$ ).

As expected, out of the linear range, the response time is much higher. For a $1600 \mu \varepsilon$ strain, the response time is as high as $19 \mathrm{~s}$; it appears that from about $1200 \mu \varepsilon$, the device cannot follow the increase in strain, thus suggesting a settling effect in the CNN at larger strain levels.
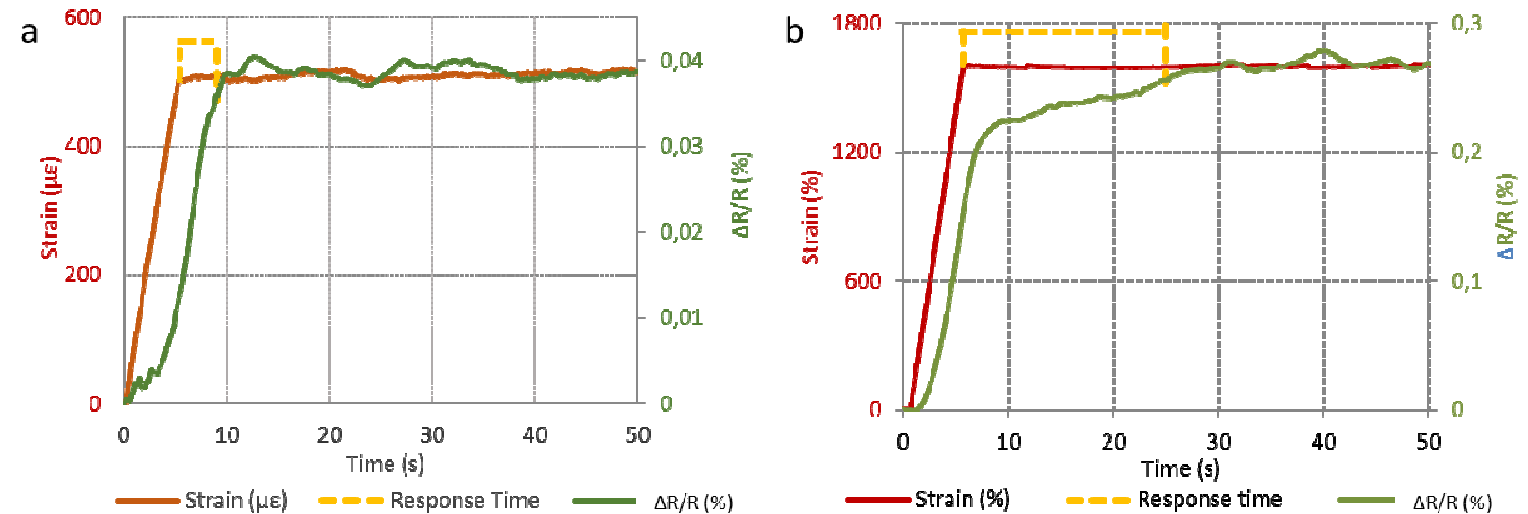

Figure 6 Measurement of device response time: a) 3.6 s under small strains $(500 \mu \varepsilon)$. b) 19 s under large strains $(1500 \mu \varepsilon)$. 


\subsection{Quasi-static cyclability and hysteresis-free operation in quasi-static mode}

We evaluated the quasi-static cyclability of the devices in the linear regime. The devices are submitted to cyclic loadings ( 20 cycles over 3 hours) in the linear regime $(800 \mu \varepsilon)$ with 9 minutes periodicity ( $2 \mathrm{mHz}$, well below the frequency limit defined using the response time). The device response is acquired at $6.7 \mathrm{~Hz}$ (3600 measurements per period).

Over 3 hours and 20 cycles, the devices display remarkable cyclability and hysteresis-free operation (Figure $7 \mathrm{a}$ and $\mathrm{b}$ ). The discrepancy between gauge factor measured during loading and the one measured during unloading is only $2.7 \%$ (figure $7 a$ ), which underlines the high reversibility of the devices compared to the state of the art [48] [49]. As can be observed in Figure 7a, there is no significant a baseline drift, though there is a significant variability in the cycle min and max values (Figure $7 \mathrm{~b})$. The standard deviation over the maximum resistance value $\left(0.58 \times 10^{-2}\right)$ is $4.7 \%$ over 20 cycles (range $\left.0.55-0.61 \times 10^{-2}\right)$. Based on the thermal sensitivity of the devices $\left(-1.0 \times 10^{-3}\right)$, a relative resistance variation of $6 \times 10^{-4}$ can be explained by only $0.6^{\circ} \mathrm{C}$ of thermal variation. This is the range of magnitude of temperature variations (between $0.3^{\circ} \mathrm{C}$ and $1^{\circ} \mathrm{C}$ ) measured in the climate-controlled room where the experiments were carried out. Hence, the variability in the resistance/strain curves is attributed to temperature variations.

Hysteresis free operation is possible only in the linear regime of the devices. Additional resistancestress curves are provided in SI9 for strains up to $2500 \mu \varepsilon$ and up to $4000 \mu \varepsilon$. In this range of strain, the devices clearly display hysteresis and creep effects. The creep effect on the resistance can be attributed to the creep of the substrate, estimated at $27 \mu \varepsilon$ by cycle for $8 \mathrm{~N}$ load cycles with 5 min periodicity.
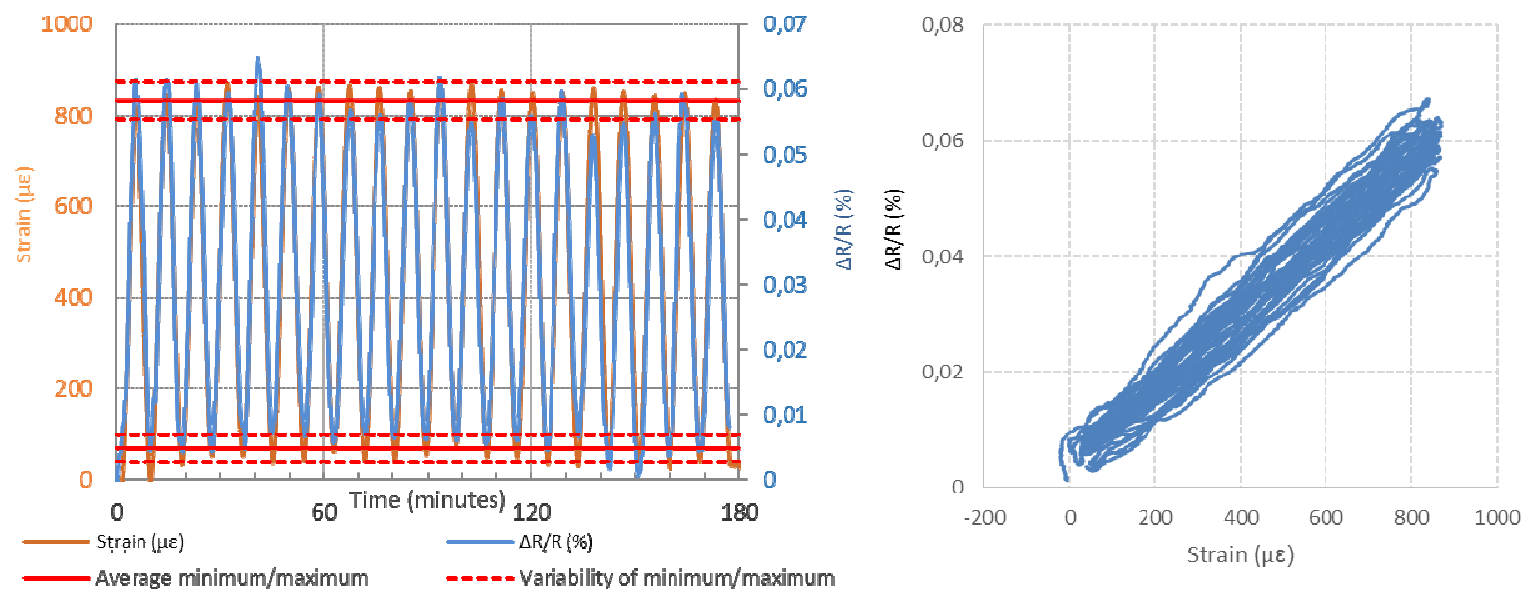

Figure 7 a) Response of a device to 20 cycles of strains between 0 and $800 \mu \varepsilon$. No evident hysteresis or baseline drift is observed. b) Corresponding resistance-strain plot. The extent of the bundle of resistance-strain curve is due to a slight 
variability (4.7\%) in $\min$ and max resistance value. It is attributed to temperature variations in the range of approx. $\pm 0.3^{\circ} \mathrm{C}$.

\section{Conclusions}

In this paper, we study for the first time the sensitivity variability of $\mathrm{CNN}$-based flexible ohmic sensors. We focus on CNN-based strain gauges on Ethylene Tetrafluoroethylene sheets achieved by inkjet printing of MWCNTs dispersed in Dichlorobenzene with SDBS as a surfactant. The use of inkjet printing produces highly reproducible devices with low variability in resistance (best standard deviation $8.4 \%$ for a 3-device batch; 20\% standard deviation averagely between 5 batches between 3 and 72 devices), gauge factor ( $16 \%$ standard deviation for a 8-device batch with $15 \%$ standard deviation on the resistance), and temperature sensitivity ( $8 \%$ standard deviation for a 8-device batch with $15 \%$ standard deviation on the resistance). Compared to the state of the art of CNN strain gauges, the devices demonstrate remarkable cyclability and hysteresis-free operation. These results open the road towards the use of the proposed sensors in real-life applications.

\section{Acknowledgments}

The authors acknowledge support from ANR (France) for project Sense-City (ANR-10-EQPX-48). The authors also thank Costel -Sorin Cojocaru, Denis Tondelier, Eléonor Caristan and Jean-Luc Sorin from LPICM and IFSTTAR laboratories for their help and support.

\section{Bibliography}

[1] A. Peigney, C. Laurent, E. Flahaut, R. Bacsa and A. Rousset, "Specific surface area of carbon nanotubes and bundles of carbon nanotubes," Carbon, vol. 39, no. 4, pp. 507-514, 2001.

[2] R. H. Baughman, A. A. Zakhidov and W. A. d. Heer, "Carbon Nanotubes-the Route Toward Applications," Science, vol. 297, p. 787, 2002.

[3] J. Kong, N. R. Franklin, C. Zhou, M. G. Chapline, S. Peng, K. Cho and H. Dai, "Nanotube Molecular Wires as Chemical Sensors," Science, vol. 287, p. 622, 2000.

[4] Y. Li, Y. Zhao, Y. Zhu, J. Rodriguez, J. Morante, E. Mendozac, C. Poa and S. Silva, "Mechanical and $\mathrm{NH} 3$ sensing properties of long multi-walled carbon nanotube ropes," Carbon, vol. 44, no. 9, pp. 1821-1825, 2006.

[5] P. W. Barone, S. B. D. A. Heller and M. S. Strano, "Near-infrared optical sensors based on singlewalled carbon nanotubes," Nature Materials, vol. 4, pp. 86-92, 2005.

[6] K. Bradley, J.-C. P. Gabriel and G. Grüner, "Flexible Nanotube Electronics," Nano Letters, vol. 3, no. 10, pp. 1353-1355, 2003. 
[7] M. Mabrook, C. Pearson, A. Jombert, D. Zeze and M. Petty, "The morphology, electrical conductivity and vapour sensing ability of inkjet-printed thin films of single-wall carbon nanotubes," Carbon, vol. 47, no. 3, pp. 752-757, 2009.

[8] T. Yamada, Y. Hayamizu, Y. Yamamoto, Y. Yomogida, A. Izadi-Najafabadi, D. N. Futaba and K. Hata, "A stretchable carbon nanotube strain sensor for human-motion detection," Nature Nanotechnology, vol. 6, pp. 296-301, 2011.

[9] S. Laflamme, M. Kollosche, J. Connor and G. Kofod, "Robust Flexible Capacitive Surface Sensor for Structural Health Monitoring Applications," Journal of Engineering Mechanics, vol. 139, no. 7, pp. 879-885, 2013.

[10] K. J. Loh, J. Kim, J. P. Lynch, N. W. S. Kam and N. A. Kotov, "Multifunctional layer-by-layer carbon nanotube-polyelectrolyte thin films for strain and corrosion sensing," Smart Materials and Structures, vol. 16, pp. 429-438, 2007.

[11] M. S. Mauter and M. Elimelech, "Environmental Applications of Carbon-Based Nanomaterials," Environmental Science and Technology, vol. 42, no. 16, pp. 5843-5859, 2008.

[12] M.-F. Yu, O. Lourie, M. J. Dyer and K. Moloni, "Strength and Breaking Mechanism of Multiwalled Carbon Nanotubes Under Tensile Load," Science, vol. 287, p. 637, 2000.

[13] A. Saha, C. Jiang and A. A. Martí, "Carbon nanotube networks on different platforms," Carbon, vol. 79, pp. 1-18, 2014.

[14] Y. Li and M. Kröger, "A theoretical evaluation of the effects of carbon nanotube entanglement and bundling on the structural and mechanical properties of buckypaper," Carbon, vol. 50, no. 5, pp. 1793-1806, 2012.

[15] D. J. Lipomi, M. Vosgueritchian, B. C.-K. Tee, S. L. Hellstrom, J. A. Lee, C. H. Fox and Z. Bao, "Skinlike pressure and strain sensors based on transparent elastic films of carbon nanotubes," Nature Nanotechnology, vol. 6, pp. 788-792, 2011.

[16] J. Noh, M. Jung, K. Jung, G. Lee, S. Lim, D. Kim, S. Kim, J. M. Tour and G. Cho, "Integrable single walled carbon nanotube (SWNT) network based thin film transistors using roll-to-roll gravure and inkjet," Organic Electronics, vol. 12, no. 12, pp. 2185-2191, 2011.

[17] P. H. Lau, K. Takei, C. Wang, Y. Ju, J. Kim, Z. Yu, T. Takahashi, G. Cho and A. Javey, "Fully Printed, High Performance Carbon Nanotube Thin-Film Transistors on Flexible Substrates," Nano Letters, vol. 13, no. 8, pp. 3864-3869, 2013.

[18] S. Li, J. G. Park, S. Wang, R. Liang, C. Zhang and B. Wang, "Working mechanisms of strain sensors utilizing aligned carbon nanotube network and aerosol jet printed electrodes," Carbon, vol. 73, pp. 303-309, 2014.

[19] A. Lesch, F. Cortés-Salazar, M. Prudent, J. Delobel, S. Rastgar, N. Lion, J.-D. Tissot, P. Tacchini and H. H. Girault, "Large scale inkjet-printing of carbon nanotubes electrodes for antioxidant assays in blood bags," Journal of Electroanalytical Chemisty, Vols. 717-718, pp. 61-68, 2014.

[20] D. J. Cohen, D. Mitra, K. Peterson and M. M. Maharbiz, "A Highly Elastic, Capacitive Strain Gauge Based on Percolating Nanotube Networks," Nano Letters, vol. 12, pp. 1821-1825, 2012. 
[21] F. Rigoni, G. Drera, S. Pagliara and A. Goldoni, "High sensitivity, moisture selective, ammonia gas sensors based on single-walled carbon nanotubes functionalized with indium tin oxide nanoparticles," Carbon, vol. 80, pp. 356-363, 2014.

[22] A. Gohier, A. Dhar, L. Gorintin, P. Bondavalli, Y. Bonnassieux and C. S. Cojocaru, "All-printed infrared sensor based on multiwalled carbon nanotubes," Applied Physics Letters, vol. 98, p. 063103, 2011.

[23] J. M. A. Katherine A. Mirica, J. G. Weis, J. M. Schnorr and T. M. Swager, "Rapid prototyping of carbon-based chemiresistive gas sensors on paper," PNAS, pp. 3265-3270, 2013.

[24] B. Y. Lee, M. G. Sung, J. Lee, K. Y. Baik, Y.-K. Kwon, M.-S. Lee and S. Hong, "Universal Parameters for Carbon Nanotube Network-Based Sensors: Can Nanotube Sensors Be Reproducible?," ACS Nano, vol. 5, no. 6, pp. 4373-4379, 2011.

[25] C. Wang, J.-C. Chien, K. Takei, T. Takahashi and J. Nah, "Extremely Bendable, High-Performance Integrated Circuits Using Semiconducting Carbon Nanotube Networks for Digital, Analog, and Radio-Frequency Applications," Nano Letters, vol. 12, pp. 1527-1533, 2012.

[26] S. Kim, T. Kim, Y. Kim, H. Choi, H. Lim, S. Yang and C. Park, "Surface modifications for the effective dispersion of carbon nanotubes in solvents and polymers," Carbon, vol. 50, no. 1, pp. 333, 2012.

[27] A. Benchirouf, E. Sowade, A. Al-Hamry, T. Blaudeck, O. Kanoun and R. Baumann, "Investigation of RFID passive strain sensors based on carbon nanotubes using inkjet printing technology," in 9th IEEE Int. Multiconference on Systems, Signals and Devices, 2012.

[28] K. S. Karimov, F. A. Khalid and M. T. S. Chani, "Carbon nanotubes based strain sensors," Measurement, vol. 45, pp. 918-921, 2012.

[29] J. Kim, J.-H. Yun, J.-W. Song and C.-S. Han, "The spontaneous metal-sitting structure on carbon nanotube arrays positioned by inkjet printing for wafer-scale production of high sensitive gas sensor units," Sensors and Actuators B: Chemical, vol. 135, pp. 587-591, 2009.

[30] K. A. Mirica, J. M. Azzarelli, J. G. Weis, J. M. Schnorr and T. M. Swager, "Rapid prototyping of carbon-based chemiresistive gas sensors on paper," PNAS, pp. 3265-3270, 2013.

[31] J. Li, Y. Lu, Q. Ye, M. Cinke, J. Han and M. Meyyappan, "Carbon Nanotube Sensors for Gas and Organic Vapor Detection," Nano Letters, vol. 3, pp. 929-933, 2003.

[32] B. Y. Lee, M. G. Sung, J. Lee, K. Y. Baik, Y.-K. Kwon, M.-S. Lee and S. Hong, "Universal Parameters for Carbon Nanotube Network-Based Sensors: Can Nanotube Sensors Be Reproducible?," ACS Nano, vol. 5, pp. 4373-4379, 2011.

[33] E. P. DeGarmo, J. T. Black and R. A. Kohser, Materials and Processes in Manufacturing, Wiley, 2003.

[34] P. Slobodian, P. Riha, A. Lengalova, R. Olejnik and D. Kimmer, "Effect of compressive strain on electric resistance of multi-wall carbon nanotube networks," Journal of Experimental Nanoscience, vol. 6, no. 3, p. 294-304, 2011. 
[35] O.-S. Kwon, H. Kim, H. Ko, J. Lee, B. Lee and C.-H. Jung, "Fabrication and characterization of inkjet-printed carbon nanotube electrode patterns on paper," Carbon, vol. 58, pp. 116-127, 2013.

[36] A. A. Cottey, "The electrical conductivity of thin metal films with very smooth surfaces," Thin Solid Films, vol. 1, no. 4, p. 297-307, 1938.

[37] H. Hoffmann and J. Vancea, "Critical assessment of thickness-dependent conductivity of thin metal films," Thin Solid Films, vol. 85, no. 2, p. 147-167, 1981.

[38] F. J. Baeza, O. Galao, E. Zornoza and E. Garcés, "Multifunctional Cement Composites Strain and Damage Sensors Applied on Reinforced Concrete Structural Elements," Materials, vol. 6, pp. 841-855, 2013.

[39] T. Kim, J. Byun, H. Song and Y. Hong, "Inkjet-printed SWCNT films for stretchable electrode and strain sensor applications," in Device Research Conference, 2012.

[40] E. Omega, "Strain gauge technical data sheet".

[41] I. Tokyo Sokki Kenkyujo Co., "Strain Gauge Performance Characteristics," 2011.

[42] N. Hu, Y. Karube, M. Arai, T. Watanabe and C. Yan, "Investigation on sensitivity of a polymer/carbon nanotube composite strain sensor," Carbon, vol. 48, pp. 680-687, 2010.

[43] S. Dehghani, M. K. Moravvej-Farshi and M. H. Sheikhi, "Temperature Dependence of Electrical Resistance of Individual Carbon Nanotubes and Carbon Nanotubes Network," Modern Physics Letters B, vol. 26, no. 21, 2012.

[44] Fisher | Moore, "Ethylene tetrafluoroethylene (ETFE) Technical Information," 1999.

[45] W. S. Bao, S. A. Meguid, Z. Z. H. and G. Zeng, "Tunneling resistance and its effect on the electrical conductivity of carbon nanotube nanocomposites," Journal of Applied Physics, vol. 111, no. 093726, 2012.

[46] V. Scardaci, R. Coull, J. Coleman, L. Byrne and G. Scott, "Carbon Nanotube network based sensors," in Conference on Nanotechnology IEEE-Nano, Birmingham, 2012.

[47] "Copolymère d'Ethylène-tetrafluoroéthylène (ETFE) - Informations Matériau," [Online]. Available: http://www.goodfellow.com/F/Copolymere-d'Ethylene-tetrafluoroethylene.html.

[48] R. Zhang, H. Deng, R. Valenca, J. Jin, Q. Fu, E. Bilotti and T. Peijs, "Strain sensing behaviour of elastomeric composite films containing carbon nanotubes under cyclic loading," Composites Science and Technology, vol. 74, no. 24, pp. 1-5, 2013.

[49] R. K. Srivastava, V. S. M. Vemuru, Y. Zeng, R. Vajtai, S. Nagarajaiah, P. M. Ajayan and A. Srivastava, "The strain sensing and thermal-mechanical behavior of flexible multi-walled carbon nanotube/polystyrene composite films," Carbon, vol. 49, no. 12, pp. 3928-3936, 2011.

[50] A. Benchirouf, E. Sowade, A. Al-Hamry, T. Blaudeck, O. Kanoun and R. Baumann, "Investigation of RFID passive strain sensors based on carbon nanotubes using inkjet printing technology," in 9th International Multiconference on Systems, Signals and Devices, 2012. 
